\title{
A New Metric of System Resource Utilization for WCDMA Systems
}

\author{
Jia-Chun Kuo and Wanjiun Liao \\ Department of Electrical Engineering \\ National Taiwan University \\ Taipei, Taiwan \\ Email:wjliao@cc.ee.ntu.edu.tw
}

\begin{abstract}
In this paper, we suggest a new measure for system resource consumed in each transmission so as to improve the overall performance of WCDMA systems. In existing discussions of resource allocation for WCDMA, they only consider "time slots" or "transmission rates" are considered, but ignore "power", a valuable resource in WCDMA systems. In WCDMA, the total power level of all users needs to be maintained under a certain threshold. However, a higher transmission rate needs a higher power level. This conflict between the transmission rate and the power level renders existing resource allocation schemes inadequate. In this paper, we define a new metric to capture the system resource consumed in one transmission and propose a credit-based fair scheduling mechanism based on the defined new metric. We verify this definition with some existing adaptation schemes and also provide simulation results to show the fairness among multiple users using the proposed credit-based fair scheduling mechanism.
\end{abstract}

Keywords: WCDMA, power, metric, fair scheduling

\section{Introduction}

\footnotetext{
The Wideband Code Division Multiple Access (WCDMA) system is an interference-limited system. In other words, the total power level from all users in a cell has to be controlled under a certain threshold. As such,
}

users can be prevented from severe power interference. On the other hand, the transmission rate of each user is proportional to the power level allowed to use. The higher the data rate, the higher the power level needed. As a result, users may use excessive power level to reach the desired data rates in the absence of arbitration. In the literature, many issues relating to fair scheduling [1-7] and users' power constraints have been studied [8]. However, little attention has been paid to discuss the conflict between the transmission rate and the power level. In this paper, we discuss the resource management for the WCDMA system under which these two trade-offs are considered. We then define a new metric of system resource utilization for WCDMA systems and propose a credit-based fair scheduling mechanism based on the new defined metric.

The rest of the paper is organized as follows. In Sec. 2, the new metric is defined, and verified with existing adaptation schemes. In Sec. 3, a credit-based fair scheduling using the newly defined metric is proposed. The simulation results are also provided to show the performance. Finally, the paper is concluded in Sec. IV.

\section{New Metric of System Utilization}

To improve the resource utilization of the 


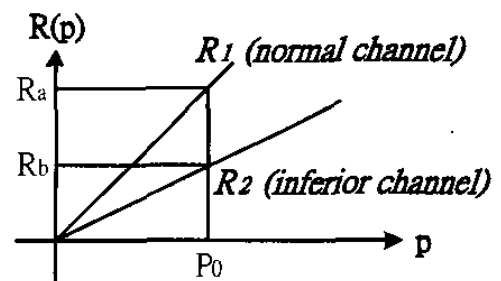

Figure 1. Rate adaptation scheme system under such conflicting constraints, we revisit the definition of system resource in the WCDMA system. Traditionally, resource allocation is primarily concerned only with "time slot" or "rate" allocation. One research attempt is to make all users fairly (or proportionally) share the system resource defined as "time slots." In other words, "bandwidth" is the only important system resource. This definition may work in wired networks, but may not be appropriate in the WCDMA system, because "power" is also a valuable resource in the WCDMA system.

In this paper, we consider both user's transmission rate and the power level in resource allocation. To correlate the transmission rate with the corresponding power level, we define a new metric $C=\int_{0}^{L} R(p) d p$ to indicate how much system resource is consumed in one transmission. Here $R(p)$ is a function to determine the transmission rate of a user when its power level is at $p$, and $\mathrm{L}$ is the power level used in one transmission. This metric can reflect the usage of the system resource more accurately in many transmission schemes and can also serve as a measurement parameter for resource management in WCDMA.

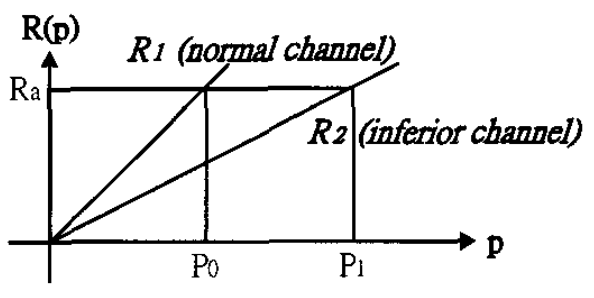

Figure 2. Power adaptation scheme

To verify our new definition, we consider two adaptation schemes in a system with two channel states: a normal channel state (and the corresponding transmission function is $R I$ ) and an inferior one (and the corresponding transmission function is $R 2$ ). We assume that the slope of $R 2$ is half that of $R 1$. Fig. 1 shows the rate adaptation scheme, in which the power level of a user is fixed at a constant level and the transmission rate is changed according to different channel states. From Fig. 1, we observe that if the power level of a user is always fixed at $\mathrm{P} 0$, the user will have a lower data rate in the inferior channel state (e.g., $\mathrm{Ra}$ is half of $\mathrm{Rb}$ ), thus also having a smaller $C$ value $(C$ value for $R b$ is half that of Ra). Fig. 2 shows another scheme called the "power adaptation scheme," in which the power level of a user is adapted to different states so as to maintain the transmission rate at a constant level. From Fig. 2, we see that the user's power level has to be increased from P0 to P1 to maintain a constant transmission rate $\mathrm{Ra}$, and the $C$ value for the user in the inferior channel state is twice that in the normal channel state.

Based on our new definition of system resource, we claim that the power adaptation scheme consumes more system resource than the rate adaptation scheme, because the former has a 
larger $\mathrm{C}$ value than the latter. This conclusion is intuitively satisfying because power adaptation is originally designed for those users which need more system resources (e.g., real-time applications) and the rate adaptation is designed for those which need less resources (e.g., non real-time applications).

\section{Credit-based Fair Scheduling Scheme}

The new definition of the system resource consumed by users (i.e., the $\mathrm{C}$ value) can be used as a new metric for many different resource management technologies. For example, we could use this $C$ value as the basic unit in the fair scheduling mechanism for WCDMA systems, instead of the time slot or transmission rate as in traditional mechanisms. To show how it works, we demonstrate a credit-based scheduling algorithm based on the proposed definition. The detailed operation is summarized as follows.

\section{Algorithm}

Initialization phase

$\forall l, l \in\{1, \ldots . N\}$, Set $C_{l} \leftarrow 0$

\section{Operation}

1) Choose those queues $\left\{l_{1}, \ldots, l_{k}\right\}$ which are backlogged;

2) Update

$$
C_{l_{i}} \leftarrow \min \left\{C_{l_{i}}+D, B\right\} \quad,
$$

$i \in\{1, \ldots, k\}$ and $\mathrm{B}$ is a bound on credit value

3) while any possible transmission schemes existing in all queues do

(i) Choose the queue $l$ which has the biggest value $C_{l}, i \in\{1, \ldots, k\}$;

(ii) According to the channel state, assign proper transmission power $P_{l}$, then get the corresponding data rate $R_{l}$, and let queue $l$ use this scheme for transmission

$$
\text { (iii) Update } C_{l} \leftarrow C_{l}-\int_{0}^{L} R(p) d p
$$

\section{4) end while}

5) Goto step 1)

In this algorithm, we use the value $\mathrm{C}$ as the credit unit, rather than the transmission rate. As in all credit-based mechanisms, the unused credit can be accumulated for future use. Each user is associated with three parameters, i.e., a credit, a usage, and an excess, where excess = usage credit. The one with the least excess value can transmit first.

We also perform simulations to evaluate the performance of the credit-based mechanism. Ten different users compete to access the same channel. Fig. 3 shows that the faimess bound of the credit-based algorithm is limited under different inter-arrival time and different service time. In other words, the fairness is maintained.

Fig. 4 shows the average throughput of ten different users. We see that the ten users have the same average throughput even when the power level is also taken into accounts. This proves that all users can fairly share the resource in WCDMA systems.

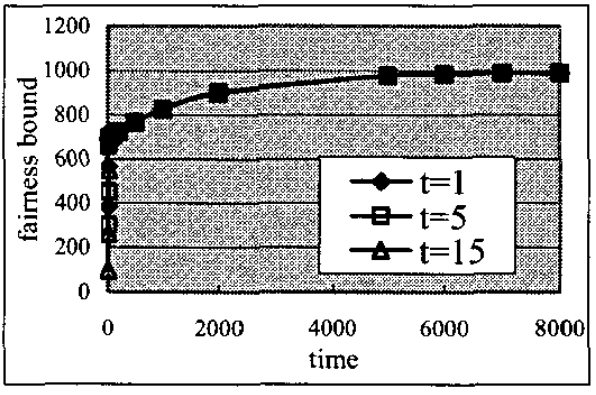

(a) Inter-arrival time 


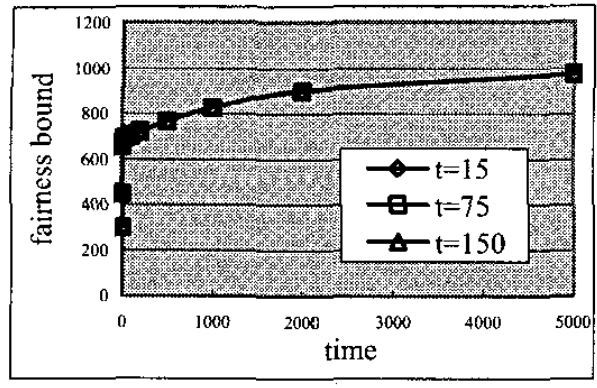

(b) Service time

Figure 3. Fairness bounds

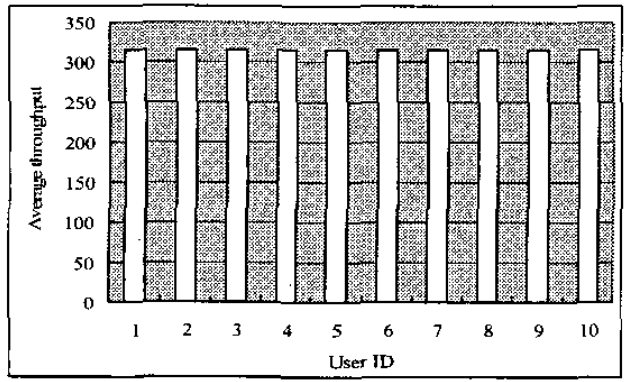

Figure 4. Average throughput of each user

\section{Conclusions}

In this paper, we suggest a new criterion of system resource for resource management in WCDMA systems. We verify this definition with some existing adaptation schemes and further demonstrate how to use our new definition in the credit-based fair scheduling mechanism to properly allocate resource allocation. From the simulation results, we show the fairness among multiple users using the proposed credit-based fair scheduling algorithm is maintained and all users still have the same average throughput.

\section{Acknowledgement}

This work was supported by the National Science Council, Taiwan, under grant number NCS92-2213-E-002-013.

\section{References}

[1] Y. Cao and V. O. K. Li, "Scheduling Algorithms in Broad-Band Wireless Networks," Proc. of the IEEE, Vol.89, No.1, Jan. 2001.

[2] B. Bensaou, D. H. K. Tsang, and K. T. Chan, "Credit-Based Fair Queueing (CBFQ): A Simple Service-Scheduling Algorithm for Packet-Switched Networks," IEEE/ACM Trans. on Networking, vol.9, no.5, Oct. 2001.

[3] S. Lu, V. Bharghavan and R. Srikant, "Fair Scheduling in Wireless Packet Networks," IEEE/ACM Trans. On Networking, vol.7, no.4, Aug. 1999.

[4] V. Bharghavan, S. Lu and T. Nandagopal, "Fair Queuing in Wireless Networks: Issues and Approaches," IEEE Personal Communications, pp.44-53, Feb. 1999.

[5] L. Wang, Y. Kwok, W. Lau and V. K. N. Lau, "Channel Capacity Fair Queueing in Wireless Networks: Issues and A New Algorithm," in Proc. IEEE ICC'2002, pp.3116-3120.

[6] I. Koutsopoulos and L. Tassiulas, "Channel state-Adaptive techniques for Throughput Enhancement in Wireless Broadband Networks," in Proc. IEEE INFOCOM'2001, pp.757-766.

[7] D.A. Eckhardt and P. Steenkiste, "Effort-limited Fair (ELF) Scheduling for Wireless Networks," in Proc. IEEE INFOCOM'2000, pp.1097-1106.

[8] S. W. Kim and Y. H. Lee, "Combined Rate and Power Adaptation in DS/CDMA Communications over Nakagami Fading Channels," IEEE Trans. on. Comm, vol.48, no. 1 , Jan. 2000. 